\title{
Vibration of orthotropic doubly curved panel with a set of inclusions of arbitrary configuration with different types of connections with the panel
}

\author{
Shopa T. \\ Pidstryhach Institute for Applied Problems of Mechanics and Mathematics \\ of the National Academy of Sciences of Ukraine, \\ $3 b$ Naukova Str., Lviv, 79060, Ukraine
}

(Received 7 September 2018)

\begin{abstract}
In the framework of the refined theory of shells, which takes into account transverse shear deformation and all inertial components, the solution of the problem on the steady-state vibration of the orthotropic doubly curved panel with the arbitrary number of absolutely rigid inclusions of the arbitrary geometrical form and location is constructed. The inclusions have different types of connections with the panel and perform the translational motion in the normal direction to the middle surface of the panel. The external boundary of the panel is of the arbitrary geometrical configuration. The arbitrary mixed, harmonic in time, boundary conditions are considered on the external boundary of the panel. The solution is built on the basis of the indirect boundary elements method. The sequential approach to the representation of the Green's functions is used. The integral equations are solved by the collocation method.
\end{abstract}

Keywords: orthotropic doubly curved panel, inclusions, vibration, indirect boundary elements method.

2000 MSC: $74 \mathrm{H} 45$

UDC: 539.3

DOI: $10.23939 / \mathrm{mmc} 2018.02 .221$

\section{Introduction}

The vibration of shells and plates with the attached masses or the concentrated masses at the points received much attention among researchers. There are also works known in literature dealing with the dynamic problems for the 3-D infinite elastic body with the inclusions [1-5]. However, to the author's knowledge, there is a scarcity of published materials regarding vibration of the finite curved panels carrying the massive inclusions of some shape penetrating the panel through all its thickness. Solutions of the problems on the steady-state vibration of the orthotropic plate and cylindrical panel with absolutely rigid inclusion rigidly fixed of the arbitrary shape and location are built $[6,7]$. The problems on the steady-state vibration of the orthotropic doubly curved panels with the arbitrary number of inclusions of the arbitrary shapes and locations interacting with the panel via the thin elastic Winkler-type interlayers, rigidly connected with panel or simply supported are considered in [7-10]. The purpose of the present work is to construct the solution in framework of the first-order shear deformation theory of shells for the problem on the vibration of the orthotropic doubly curved panel with any number of inclusions of any shape and location with different types of connections with the panel simultaneously, when an outer boundary of the panel has any form and the arbitrary mixed boundary conditions are imposed on it. 


\section{Problem statement}

Consider the problem on the steady-state vibration of the orthotropic doubly curved panel with totally $N$ absolutely rigid inclusions. Among them are $N_{1}$ inclusions of an arbitrary form and location interacting with the panel via thin Winkler-type elastic interlayers with the stiffness $k^{(j)}(\alpha),\left(j=\overline{1, N_{1}}\right)$, $N_{2}$ inclusions of the arbitrary form and location that are rigidly connected with the panel, and $N_{3}$ inclusions of the arbitrary form and location that are simply supported. The stiffness of the elastic interlayers can be different for each inclusion and, generally, can vary along the contours of the inclusions. The contours of the inclusions are the curves $L^{(j)}, j=\overline{1, N}$. The force with the principal vector $P^{(j)}=P_{0}^{(j)} \sin (\omega t)$ normal to the middle surface of the panel is acting on the inclusion with the mass $\tilde{m}^{(j)}$. Suppose that inclusions perform the translational motion along the normal direction to the middle surface of the panel and $\tilde{w}^{(j)}(t)=\tilde{w}_{0}^{(j)} \sin (\omega t)$ is the displacement of the $j$-th inclusion. The external boundary of the panel is also of the arbitrary form and its contour consists of three curves $L^{(N+1)}, L^{(N+2)}$ and $L^{(N+3)}$ complementing each other. The middle surface of the panel occupies the multi-connected domain $\Omega$. The domain $\Pi$ is a singly connected rectangular in plane domain with the sides of the length $l_{1}, l_{2}$, that contains the domain $\Omega$. The domain $\Pi$ is chosen in the way that its sides are directed along the principal directions of the orthotropy of the material. Coordinate lines of the curvilinear system of coordinates are directed along the sides of the rectangular in plane domain $\Pi$ and the origin is located in one of its vertices. The metrics of the curvilinear system of coordinates is considered to be close to the Euclidean.

Such notations are used: $\alpha=\left\{\alpha_{1}, \alpha_{2}\right\}$ are curvilinear coordinates, $t$ is time, $\boldsymbol{n}, \boldsymbol{\tau}$ are normal and tangential vectors along some direction; $E_{i}$ is Young's moduli; $G_{12}, G_{13}, G_{23}$ are shear moduli; $\nu_{12}, \nu_{21}$ are Poisson coefficients; $\rho$ is the density; $k_{1}, k_{2}$ are principal curvatures of the panel; $2 h$ is the thickness of the panel; $w$ is deflection of the panel; $u_{1}, u_{2}$ are axial displacements of the points of the middle surface of the panel; $u_{n}, u_{\tau}$ are normal and tangential components of the axial displacements of the points of the middle surface of the panel; $\gamma_{1}, \gamma_{2}$ are the angles of rotation of the normal to the middle surface of the panel; $\gamma_{n}, \gamma_{\tau}$ are the normal and tangential components of the angles of rotation of the normal to the middle surface of the panel; $Q_{n}$ is the normal component of the shear force; $M_{n}, N_{n}$ are normal components of the bending moments and axial forced, $M_{\tau}, N_{\tau}$ are tangential components of the bending moments and axial forced.

The boundary conditions on the contours of the inclusions interacting with the panel via the elastic Winkler-type interlayers take the form

$$
\begin{aligned}
Q_{n}(\alpha, t)=-p^{(j)}(\alpha, t), \quad M_{n}(\alpha, t) & =0, \quad N_{n}(\alpha, t)=0, \quad M_{\tau}(\alpha, t)=0, \quad N_{\tau}(\alpha, t)=0, \\
\alpha & \in L^{(j)}, \quad j=\overline{1, N_{1}},
\end{aligned}
$$

where $p^{(j)}(\alpha, t)=-k^{(j)}(\alpha)\left(\tilde{w}^{(j)}(t)-w(\alpha, t)\right)$ are the contact forces of interaction between the panel and the $j$-th inclusion, $w(\alpha, t)$ is the deflection of the panel on the edge just before the the elastic interlayer, $k^{(j)}(\alpha)$ is the stiffness of the elastic interlayer between the $j$-th inclusion and the panel.

The boundary conditions on the contours of the inclusions rigidly connected with the panel are as follows:

$$
\begin{gathered}
w(\alpha, t)=\tilde{w}^{(j)}(t), \quad u_{n}(\alpha, t)=0, \quad \gamma_{n}(\alpha, t)=0, \quad u_{\tau}(\alpha, t)=0, \quad \gamma_{\tau}(\alpha, t)=0, \\
\alpha \in L^{(j)}, \quad j=\overline{N_{1}+1, N_{1}+N_{2}} .
\end{gathered}
$$

On the contours of the simply supported inclusions, the boundary conditions take the form

$$
\begin{gathered}
w(\alpha, t)=\tilde{w}^{(j)}(t), \quad u_{\tau}(\alpha, t)=0, \quad \gamma_{\tau}(\alpha, t)=0, \quad M_{n}(\alpha, t)=0, \quad N_{n}(\alpha, t)=0, \\
\alpha \in L^{(j)}, \quad j=\overline{N_{1}+N_{2}+1, N_{1}+N_{2}+N_{3}} .
\end{gathered}
$$


The contact forces of interaction between the panel and the rigidly connected and simply supported inclusions are modeled as follows:

$$
p^{(j)}(\alpha, t)=-Q_{n}(\alpha, t)=-Q_{n}(\alpha) \sin (\omega t), \quad \alpha \in L^{(j)}, \quad j=\overline{N_{1}+1, N_{1}+N_{2}+N_{3}} .
$$

The boundary conditions on the outer boundary of the panel are the following:

$$
\begin{gathered}
w(\alpha, t)=w_{0}^{(N+1)}(\alpha) \sin (\omega t), \quad u_{n}(\alpha, t)=u_{n 0}^{(N+1)}(\alpha) \sin (\omega t), \quad \gamma_{n}(\alpha, t)=\gamma_{n 0}^{(N+1)}(\alpha) \sin (\omega t) \\
u_{\tau}(\alpha, t)=u_{\tau 0}^{(N+1)}(\alpha) \sin (\omega t), \quad \gamma_{\tau}(\alpha, t)=\gamma_{\tau 0}^{(N+1)}(\alpha) \sin (\omega t), \quad \alpha \in L^{(N+1)} \\
Q_{n}(\alpha, t)=Q_{n 0}^{(N+2)}(\alpha) \sin (\omega t), \quad M_{n}(\alpha, t)=M_{n 0}^{(N+2)}(\alpha) \sin (\omega t), \quad N_{n}(\alpha, t)=N_{n 0}^{(N+2)}(\alpha) \sin (\omega t) \\
N_{\tau}(\alpha, t)=N_{\tau 0}^{(N+2)}(\alpha) \sin (\omega t), \quad M_{\tau}(\alpha, t)=M_{\tau 0}^{(N+2)}(\alpha) \sin (\omega t), \quad \alpha \in L^{(N+2)} \\
w(\alpha, t)=w_{0}^{(N+3)}(\alpha) \sin (\omega t), \quad M_{n}(\alpha, t)=M_{n 0}^{(N+3)}(\alpha) \sin (\omega t), \quad N_{n}(\alpha, t)=N_{n 0}^{(N+3)}(\alpha) \sin (\omega t) \\
u_{\tau}(\alpha, t)=u_{\tau 0}^{(N+3)}(\alpha) \sin (\omega t), \quad \gamma_{\tau}(\alpha, t)=\gamma_{\tau 0}^{(N+3)}(\alpha) \sin (\omega t), \quad \alpha \in L^{(N+3)}
\end{gathered}
$$

\section{Solution of the problem}

The equations of the first-order shear deformation theory of shallow shells taking into account all inertial components, including rotary inertia, are used [8-11]

$$
\begin{gathered}
{[L]\{U\}=-\{P\},} \\
\{U\}=\left\{u_{1}(\alpha, t), u_{2}(\alpha, t), w(\alpha, t), \gamma_{1}(\alpha, t), \gamma_{2}(\alpha, t)\right\}^{\mathrm{T}}, \quad\{P\}=\{0,0,0,0,0\}^{\mathrm{T}}, \\
L_{11}=B_{1} \frac{\partial^{2}}{\partial \alpha_{1}^{2}}+B_{12} \frac{\partial^{2}}{\partial \alpha_{2}^{2}}-k_{1}^{2} \Lambda_{1}-2 h \rho \frac{\partial^{2}}{\partial t^{2}}, \quad L_{22}=B_{12} \frac{\partial^{2}}{\partial \alpha_{1}^{2}}+B_{2} \frac{\partial^{2}}{\partial \alpha_{2}^{2}}-k_{2}^{2} \Lambda_{2}-2 h \rho \frac{\partial^{2}}{\partial t^{2}} \\
L_{33}=\Lambda_{1} \frac{\partial^{2}}{\partial \alpha_{1}^{2}}+\Lambda_{2} \frac{\partial^{2}}{\partial \alpha_{2}^{2}}-\left[k_{1} B_{1}\left(k_{1}+\nu_{12} k_{2}\right)+k_{2} B_{2}\left(k_{2}+\nu_{21} k_{1}\right)\right]-2 h \rho \frac{\partial^{2}}{\partial t^{2}} \\
L_{44}=D_{1} \frac{\partial^{2}}{\partial \alpha_{1}^{2}}+D_{12} \frac{\partial^{2}}{\partial \alpha_{2}^{2}}-\Lambda_{1}-\frac{2 h^{3}}{3} \rho \frac{\partial^{2}}{\partial t^{2}}, \quad L_{55}=D_{12} \frac{\partial^{2}}{\partial \alpha_{1}^{2}}+D_{2} \frac{\partial^{2}}{\partial \alpha_{2}^{2}}-\Lambda_{2}-\frac{2 h^{3}}{3} \rho \frac{\partial^{2}}{\partial t^{2}} \\
L_{14}=L_{41}=k_{1} \Lambda_{1}, \quad L_{12}=\left(B_{1} \nu_{12}+B_{12}\right) \frac{\partial^{2}}{\partial \alpha_{1} \partial \alpha_{2}}, \quad L_{21}=\left(B_{12}+B_{2} \nu_{21}\right) \frac{\partial^{2}}{\partial \alpha_{1} \partial \alpha_{2}} \\
L_{25}=L_{52}=k_{2} \Lambda_{2}, \quad L_{45}=\left(D_{1} \nu_{12}+D_{12}\right) \frac{\partial^{2}}{\partial \alpha_{1} \partial \alpha_{2}}, \quad L_{54}=\left(D_{12}+D_{2} \nu_{21}\right) \frac{\partial^{2}}{\partial \alpha_{1} \partial \alpha_{2}} \\
L_{34}=-L_{43}=\Lambda_{1} \frac{\partial}{\partial \alpha_{1}}, \quad L_{35}=-L_{53}=\Lambda_{2} \frac{\partial}{\partial \alpha_{2}}, \quad L_{24}=L_{42}=0, \quad L_{15}=L_{51}=0 \\
L_{13}=\left(k_{1} \Lambda_{1}+B_{1} k_{1}+B_{1} k_{2} \nu_{12}\right) \frac{\partial}{\partial \alpha_{1}}, \quad L_{31}=-\left(k_{1} \Lambda_{1}+B_{1} k_{1}+B_{2} k_{2} \nu_{21}\right) \frac{\partial}{\partial \alpha_{1}} \\
L_{23}=\left(k_{2} \Lambda_{2}+B_{2} k_{2}+B_{2} k_{2} \nu_{21}\right) \frac{\partial}{\partial \alpha_{2}}, \quad L_{32}=-\left(k_{2} \Lambda_{2}+B_{2} k_{2}+B_{1} k_{1} \nu_{12}\right) \frac{\partial}{\partial \alpha_{2}}
\end{gathered}
$$

The equations of translational motion of absolutely rigid inclusions take the form

$$
\tilde{m}^{(j)} \frac{\partial^{2} \tilde{w}^{(j)}(t)}{\partial t^{2}}=P^{(j)}(t)-\int_{L^{(j)}} k^{(j)}(\zeta)\left(\tilde{w}^{(j)}(t)-w(\zeta, t)\right) d l(\zeta), \quad j=\overline{1, N_{1}}
$$

Mathematical Modeling and Computing, Vol.5, No. 2, pp. 221-234 (2018) 


$$
\tilde{m}^{(j)} \frac{\partial^{2} \tilde{w}^{(j)}(t)}{\partial t^{2}}=P^{(j)}(t)-\int_{L^{(j)}} Q_{n}(\zeta, t) d l(\zeta), \quad j=\overline{N_{1}+1, N_{1}+N_{2}+N_{3}} .
$$

The solution of the boundary value problem is found on the basis of the indirect boundary elements method using the Green functions (9) for the system of partial derivative equations (7) in the rectangular in plane domain $\Pi$ with the homogeneous boundary conditions of the kind

$$
w=0, \quad M_{n}=0, \quad N_{n}=0, \quad u_{\tau}=0, \quad \gamma_{\tau}=0, \quad \alpha \in \partial \Pi,
$$

which are found in the paper [11] on the basis of the sequential approach to the representation of the Dirac delta-function and the Fourier series method $[6,12,13]$,

$$
\begin{aligned}
& \left\{U^{G}\left(\alpha, \alpha^{r}, t\right)\right\}=\lim _{\varepsilon \rightarrow 0}\left\{U^{G}\left(\alpha, \alpha^{r}, \varepsilon, t\right)\right\} \\
& =\lim _{\varepsilon \rightarrow 0} \sum_{k=0}^{\infty} \sum_{m=0}^{\infty} C_{k m}(\varepsilon)\left[E_{k m}(\alpha)\right]\left[U_{k m}\right]\left[E_{k m}\left(\alpha^{r}\right)\right]\left\{T^{r}\right\} \sin (\omega t), \\
& \left\{U^{G}\left(\alpha, \alpha^{r}, t\right)\right\}=\left\{u_{1}^{G}\left(\alpha, \alpha^{r}, t\right), u_{2}^{G}\left(\alpha, \alpha^{r}, t\right), w^{G}\left(\alpha, \alpha^{r}, t\right), \gamma_{1}^{G}\left(\alpha, \alpha^{r}, t\right), \gamma_{2}^{G}\left(\alpha, \alpha^{r}, t\right)\right\}^{\mathrm{T}}, \\
& \left\{T^{r}\right\}=\left\{T_{1}^{r}, T_{2}^{r}, T_{3}^{r}, T_{4}^{r}, T_{5}^{r}\right\}^{\mathrm{T}}, \\
& {\left[E_{k m}(\alpha)\right]=\left[\begin{array}{ccccc}
\Phi_{k m}^{c s}(\alpha) & 0 & 0 & 0 & 0 \\
0 & \Phi_{k m}^{s c}(\alpha) & 0 & 0 & 0 \\
0 & 0 & \Phi_{k m}^{s s}(\alpha) & 0 & 0 \\
0 & 0 & 0 & \Phi_{k m}^{c s}(\alpha) & 0 \\
0 & 0 & 0 & 0 & \Phi_{k m}^{s c}(\alpha)
\end{array}\right]} \\
& {\left[E_{k m}\left(\alpha^{r}\right)\right]=\left[\begin{array}{ccccc}
\Phi_{k m}^{c s}\left(\alpha^{r}\right) & 0 & 0 & 0 & 0 \\
0 & \Phi_{k m}^{s c}\left(\alpha^{r}\right) & 0 & 0 & 0 \\
0 & 0 & \Phi_{k m}^{s s}\left(\alpha^{r}\right) & 0 & 0 \\
0 & 0 & 0 & \Phi_{k m}^{c s}\left(\alpha^{r}\right) & 0 \\
0 & 0 & 0 & 0 & \Phi_{k m}^{s c}\left(\alpha^{r}\right)
\end{array}\right] \text {, }} \\
& {\left[U_{k m}\right]=\left[\begin{array}{ccccc}
u_{1 k m}^{1} & u_{1 k m}^{2} & u_{1 k m}^{3} & u_{1 k m}^{4} & u_{1 k m}^{5} \\
u_{2 k m}^{1} & u_{2 k m}^{2} & u_{2 k m}^{3} & u_{2 k m}^{4} & u_{2 k m}^{5} \\
w_{k m}^{1} & w_{k m}^{2} & w_{k m}^{3} & w_{k m}^{4} & w_{k m}^{5} \\
\gamma_{1 k m}^{1} & \gamma_{1 k m}^{2} & \gamma_{1 k m}^{3} & \gamma_{1 k m}^{4} & \gamma_{1 k m}^{5} \\
\gamma_{2 k m}^{1} & \gamma_{2 k m}^{2} & \gamma_{2 k m}^{3} & \gamma_{2 k m}^{4} & \gamma_{2 k m}^{5}
\end{array}\right]} \\
& u_{1 k m}^{1}=\frac{1}{\operatorname{det}\left(L^{k m}\right)} \operatorname{det}\left(\begin{array}{llll}
L_{22}^{k m} & L_{23}^{k m} & L_{24}^{k m} & L_{25}^{k m} \\
L_{32}^{k m} & L_{33}^{k m} & L_{34}^{k m} & L_{35}^{k m} \\
L_{42}^{k m} & L_{43}^{k m} & L_{44}^{k m} & L_{45}^{k m} \\
L_{52}^{k m} & L_{53}^{k m} & L_{54}^{k m} & L_{55}^{k m}
\end{array}\right) \text {, } \\
& u_{1 k m}^{2}=\frac{-1}{\operatorname{det}\left(L^{k m}\right)} \operatorname{det}\left(\begin{array}{llll}
L_{21}^{k m} & L_{23}^{k m} & L_{24}^{k m} & L_{25}^{k m} \\
L_{31}^{k m} & L_{33}^{k m} & L_{34}^{k m} & L_{35}^{k m} \\
L_{41}^{k m} & L_{43}^{k m} & L_{44}^{k m} & L_{45}^{k m} \\
L_{51}^{k m} & L_{53}^{k m} & L_{54}^{k m} & L_{55}^{k m}
\end{array}\right), \ldots, \\
& \operatorname{det}\left(L^{k m}\right)=\operatorname{det}\left(\begin{array}{lllll}
L_{11}^{k m} & L_{12}^{k m} & L_{13}^{k m} & L_{14}^{k m} & L_{15}^{k m} \\
L_{21}^{k m} & L_{22}^{k m} & L_{23}^{k m} & L_{24}^{k m} & L_{25}^{k m} \\
L_{31}^{k m} & L_{32}^{k m} & L_{33}^{k m} & L_{34}^{k m} & L_{35}^{k m} \\
L_{41}^{k m} & L_{42}^{k m} & L_{43}^{k m} & L_{44}^{k m} & L_{45}^{k m} \\
L_{51}^{k m} & L_{52}^{k m} & L_{53}^{k m} & L_{54}^{k m} & L_{55}^{k m}
\end{array}\right) \text {, }
\end{aligned}
$$




$$
\begin{gathered}
L_{11}^{k m}=-B_{1} \lambda_{1 k}^{2}-B_{12} \lambda_{2 m}^{2}-k_{1}^{2} \Lambda_{1}+2 \rho h \omega^{2}, \quad L_{22}^{k m}=-B_{12} \lambda_{1 k}^{2}-B_{2} \lambda_{2 m}^{2}-k_{2}^{2} \Lambda_{2}+2 \rho h \omega^{2}, \\
L_{33}^{k m}=-\Lambda_{1} \lambda_{1 k}^{2}-\Lambda_{2} \lambda_{2 m}^{2}-\left[k_{1} B_{1}\left(k_{1}+\nu_{12} k_{2}\right)+k_{2} B_{2}\left(k_{2}+\nu_{21} k_{1}\right)\right]+2 \rho h \omega^{2}, \\
L_{44}^{k m}=-D_{1} \lambda_{1 k}^{2}-D_{12} \lambda_{2 m}^{2}-\Lambda_{1}+\frac{2 h^{3}}{3} \rho \omega^{2}, \quad L_{55}^{k m}=-D_{12} \lambda_{1 k}^{2}-D_{2} \lambda_{2 m}^{2}-\Lambda_{2}+\frac{2 h^{3}}{3} \rho \omega^{2}, \\
L_{14}^{k m}=L_{41}^{k m}=k_{1} \Lambda_{1}, \quad L_{25}^{k m}=L_{52}^{k m}=k_{2} \Lambda_{2}, \quad L_{15}^{k m}=L_{51}^{k m}=L_{24}^{k m}=L_{42}^{k m}=0, \\
L_{12}^{k m}=-\left(B_{1} \nu_{12}+B_{12}\right) \lambda_{1 k} \lambda_{2 m}, \quad L_{21}^{k m}=-\left(B_{2} \nu_{21}+B_{12}\right) \lambda_{1 k} \lambda_{2 m}, \\
L_{34}^{k m}=L_{43}^{k m}=-\Lambda_{1} \lambda_{1 k}, \quad L_{35}^{k m}=L_{53}^{k m}=-\Lambda_{2} \lambda_{2 m}, \\
L_{54}^{k m}=-\left(D_{2} \nu_{21}+D_{12}\right) \lambda_{1 k} \lambda_{2 m}, \quad L_{45}^{k m}=-\left(D_{1} \nu_{12}+D_{12}\right) \lambda_{1 k} \lambda_{2 m}, \\
L_{23}^{k m}=\left(k_{2} \Lambda_{2}+B_{2} k_{2}+B_{2} k_{2} \nu_{21}\right) \lambda_{2 m}, \quad L_{32}^{k m}=\left(k_{2} \Lambda_{2}+B_{2} k_{2}+B_{1} k_{1} \nu_{12}\right) \lambda_{2 m}, \\
C_{k m}(\varepsilon)=B_{k m} \frac{4}{l_{1} l_{2}} \phi_{1}\left(\lambda_{1 k} \varepsilon_{1}\right) \phi_{2}\left(\lambda_{2 m} \varepsilon_{2}\right), \\
\lambda_{1 k}=\frac{k \pi}{l_{1}}, \quad \lambda_{2 m}=\frac{m \pi}{l_{2}}, \\
\Phi_{k m}^{c s}(\alpha)=\cos \left(\lambda_{1 k} \alpha_{1}\right) \sin \left(\lambda_{2 m} \alpha_{2}\right), \\
\Phi_{k m}^{s c}(\alpha)=\sin \left(\lambda_{1 k} \alpha_{1}\right) \cos \left(\lambda_{2 m} \alpha_{2}\right), \quad \mu_{k m}=\left\{\begin{array}{l}
1, \quad \text { if } k \neq 0, m \neq 0, \\
\frac{1}{2}, \quad \text { if } k=0, m \neq 0, \\
\frac{1}{2}, \quad \text { if } k \neq 0, m=0, \\
\Phi_{k m}^{s s}(\alpha)=\sin \left(\lambda_{1 k} \alpha_{1}\right) \sin \left(\lambda_{2 m} \alpha_{2}\right),
\end{array} \quad \begin{array}{l}
\frac{1}{4}, \quad \text { if } k=0, m=0, \\
\Phi_{k m}^{c c}(\alpha)=\cos \left(\lambda_{1 k} \alpha_{1}\right) \cos \left(\lambda_{2 m} \alpha_{2}\right),
\end{array}\right.
\end{gathered}
$$

$\phi_{1}\left(\lambda_{1 k} \varepsilon_{1}\right), \phi_{2}\left(\lambda_{2 m} \varepsilon_{2}\right)$ are the weight functions that define the method of generalized summation. For the simplicity $\varepsilon_{1}=\varepsilon_{2}=\varepsilon$ can be taken.

Consider the generalized contour $L=L^{(1)} \cup \ldots \cup L^{(N)} \cup L^{(N+1)} \cup L^{(N+2)} \cup L^{(N+3)}$ and such functions defined on it

$$
\begin{aligned}
& \{T(\xi)\}=\left\{T_{1}(\xi), T_{2}(\xi), T_{3}(\xi), T_{4}(\xi), T_{5}(\xi)\right\}^{\mathrm{T}} \\
& =\left\{\begin{array}{l}
\left\{T^{(1)}(\xi)\right\}=\left\{T_{1}^{(1)}(\xi), T_{2}^{(1)}(\xi), T_{3}^{(1)}(\xi), T_{4}^{(1)}(\xi), T_{5}^{(1)}(\xi)\right\}^{\mathrm{T}}, \quad \xi \in L^{(1)}, \\
\left\{T^{(2)}(\xi)\right\}=\left\{T_{1}^{(2)}(\xi), T_{2}^{(2)}(\xi), T_{3}^{(2)}(\xi), T_{4}^{(2)}(\xi), T_{5}^{(2)}(\xi)\right\}^{\mathrm{T}}, \quad \xi \in L^{(2)}, \\
\cdots \cdots \cdots \cdots \cdots \cdots \cdots \cdots \cdots \cdots \cdots \cdots \cdots \cdots \cdots \cdots \cdots \cdots \cdots \cdots \cdots \cdots \cdots \cdots \cdots \cdots \cdots \cdots \cdots \cdots \cdots \cdots \\
\left\{T^{(N+3)}(\xi)\right\}=\left\{T_{1}^{(N+3)}(\xi), T_{2}^{(N+3)}(\xi), T_{3}^{(N+3)}(\xi), T_{4}^{(N+3)}(\xi), T_{5}^{(N+3)}(\xi)\right\}^{\mathrm{T}}, \quad \xi \in L^{(N+3)} .
\end{array}\right.
\end{aligned}
$$

The solution is represented in the form of simple layer potential

$$
\begin{gathered}
\{U(\alpha, t)\}=\lim _{\varepsilon \rightarrow 0} \int_{L} \sum_{k=0}^{\infty} \sum_{m=0}^{\infty} C_{k m}(\varepsilon)\left[E_{k m}(\alpha)\right]\left[U_{k m}\right]\left[E_{k m}(\xi)\right]\{T(\xi)\} d l(\xi) \sin (\omega t), \\
\{U(\alpha, t)\}=\left\{u_{1}(\alpha, t), u_{2}(\alpha, t), w(\alpha, t), \gamma_{1}(\alpha, t), \gamma_{2}(\alpha, t)\right\}^{\mathrm{T}} .
\end{gathered}
$$

Integral equations for the boundary value problem are built using the representation of the solution (10) and boundary conditions (1)-(6). In the case of boundary conditions when forces are defined on the contours the method of the fictitious contours is used to avoid the discontinuity jump of the derivative of the simple layer potential on the boundaries. Such that, the boundary conditions on those contours can be satisfied not on the real contour, but on the shifted one by the small distance $\varepsilon$ inside the multi-connected domain considered in the problem. The curves of the shifted contours are denoted by $L^{\varepsilon(j)}$. Then taking into account the equations of motion (8), the system of $5(N+3)+N$ 
integral equations and integral relations with respect to the unknown density functions $\{T(\xi)\}$ and to the unknown displacements of the inclusions $\tilde{w}_{0}^{(j)}, j=\overline{1, N}$ obtains the form

$$
\begin{aligned}
& \left\{u_{n 0}^{(j)}(\alpha), u_{\tau 0}^{(j)}(\alpha), w_{0}^{(j)}(\alpha), \gamma_{n 0}^{(j)}(\alpha), \gamma_{\tau 0}^{(j)}(\alpha)\right\}^{\mathrm{T}}=\lim _{\varepsilon \rightarrow 0} \int_{L} \sum_{k=0}^{\infty} \sum_{m=0}^{\infty} C_{k m}(\varepsilon)\left[\Omega_{k m}^{(U)}(\alpha)\right]\left[E_{k m}(\xi)\right]\{T(\xi)\} d l(\xi), \\
& \alpha \in L^{(j)}, \quad j=\overline{N_{1}+1, N_{1}+N_{2}}, \quad j=N+1, \\
& \left\{N_{n 0}^{(j)}(\alpha), N_{\tau 0}^{(j)}(\alpha), Q_{n 0}^{(j)}(\alpha), M_{n 0}^{(j)}(\alpha), M_{\tau 0}^{(j)}(\alpha)\right\}^{\mathrm{T}} \\
& =\lim _{\varepsilon \rightarrow 0} \int_{L} \sum_{k=0}^{\infty} \sum_{m=0}^{\infty} C_{k m}(\varepsilon)\left[\Omega_{k m}^{(P)}(\alpha)\right]\left[E_{k m}(\xi)\right]\{T(\xi)\} d l(\xi), \quad \alpha \in L^{\varepsilon(j)}, \quad j=\overline{1, N_{1}}, \quad j=N+2, \\
& w_{0}^{(j)}(\alpha)=\lim _{\varepsilon \rightarrow 0} \int_{L} \sum_{k=0}^{\infty} \sum_{m=0}^{\infty} \sum_{i=1}^{5} C_{k m}(\varepsilon) w_{i}(\alpha) \Phi_{k m}^{i}(\xi) T_{i}(\xi) d l(\xi), \\
& u_{\tau 0}^{(j)}(\alpha)=\lim _{\varepsilon \rightarrow 0} \int_{L} \sum_{k=0}^{\infty} \sum_{m=0}^{\infty} \sum_{i=1}^{5} C_{k m}(\varepsilon) u_{i \tau}(\alpha) \Phi_{k m}^{i}(\xi) T_{i}(\xi) d l(\xi) \\
& \gamma_{\tau 0}^{(j)}(\alpha)=\lim _{\varepsilon \rightarrow 0} \int_{L} \sum_{k=0}^{\infty} \sum_{m=0}^{\infty} \sum_{i=1}^{5} C_{k m}(\varepsilon) \gamma_{i \tau}(\alpha) \Phi_{k m}^{i}(\xi) T_{i}(\xi) d l(\xi) \\
& \alpha \in L^{(j)}, \quad j=\overline{N_{1}+N_{2}, N}, \quad j=N+3, \\
& M_{n 0}^{(j)}(\alpha)=\lim _{\varepsilon \rightarrow 0} \int_{L} \sum_{k=0}^{\infty} \sum_{m=0}^{\infty} \sum_{i=1}^{5} C_{k m}(\varepsilon) M_{i n}(\alpha) \Phi_{k m}^{i}(\xi) T_{i}(\xi) d l(\xi), \\
& N_{n 0}^{(j)}(\alpha)=\lim _{\varepsilon \rightarrow 0} \int_{L} \sum_{k=0}^{\infty} \sum_{m=0}^{\infty} \sum_{i=1}^{5} C_{k m}(\varepsilon) N_{i n}(\alpha) \Phi_{k m}^{i}(\xi) T_{i}(\xi) d l(\xi) \\
& \alpha \in L^{\varepsilon(j)}, \quad j=\overline{N_{1}+N_{2}, N}, \quad j=N+3, \\
& -\omega^{2} \tilde{m}^{(j)} \tilde{w}_{0}^{(j)}=P_{0}^{(j)}-\int_{L^{(j)}} k^{(j)}(\zeta)\left(\tilde{w}_{0}^{(j)}-w(\zeta)\right) d l(\zeta), \quad j=\overline{1, N_{1}}, \\
& -\omega^{2} \tilde{m}^{(j)} \tilde{w}_{0}^{(j)}=P_{0}^{(j)}-\int_{L^{\varepsilon(j)}} Q_{n}(\zeta) d l(\zeta), \quad j=\overline{N_{1}+1, N_{1}+N_{2}+N_{3}},
\end{aligned}
$$

where

$$
\begin{gathered}
u_{n 0}^{(j)}(\alpha)=u_{\tau 0}^{(j)}(\alpha)=\gamma_{n 0}^{(j)}(\alpha)=\gamma_{\tau 0}^{(j)}(\alpha)=0, \quad w_{0}^{(j)}(\alpha)=\tilde{w}_{0}^{(j)}, \quad j=\overline{N_{1}+1, N_{1}+N_{2}}, \\
N_{n 0}^{(j)}(\alpha)=N_{\tau 0}^{(j)}(\alpha)=M_{n 0}^{(j)}(\alpha)=M_{\tau 0}^{(j)}(\alpha)=0, \quad Q_{n 0}^{(j)}(\alpha)=k^{(j)}(\alpha)\left(\tilde{w}_{0}^{(j)}-w(\alpha)\right), \quad j=\overline{1, N_{1}} \\
N_{n 0}^{(j)}(\alpha)=u_{\tau 0}^{(j)}(\alpha)=M_{n 0}^{(j)}(\alpha)=\gamma_{\tau 0}^{(j)}(\alpha)=0, \quad w_{0}^{(j)}(\alpha)=\tilde{w}_{0}^{(j)}, \quad j=\overline{N_{1}+N_{2}+1, N_{1}+N_{2}+N_{3}}, \\
w(\zeta)=\lim _{\varepsilon \rightarrow 0} \int_{L} \sum_{k=0}^{\infty} \sum_{m=0}^{\infty} \sum_{i=1}^{5} C_{k m}(\varepsilon) w_{i}(\zeta) \Phi_{k m}^{i}(\xi) T_{i}(\xi) d l(\xi), \\
w(\alpha)=\lim _{\varepsilon \rightarrow 0} \int_{L} \sum_{k=0}^{\infty} \sum_{m=0}^{\infty} \sum_{i=1}^{5} C_{k m}(\varepsilon) w_{i}(\alpha) \Phi_{k m}^{i}(\xi) T_{i}(\xi) d l(\xi),
\end{gathered}
$$

Mathematical Modeling and Computing, Vol.5, No. 2, pp. 221-234 (2018) 


$$
\begin{aligned}
& Q_{n}(\zeta)=\lim _{\varepsilon \rightarrow 0} \int_{L} \sum_{k=0}^{\infty} \sum_{m=0}^{\infty} \sum_{i=1}^{5} C_{k m}(\varepsilon) Q_{i n}(\zeta) \Phi_{k m}^{i}(\xi) T_{i}(\xi) d l(\xi), \\
& \Phi_{k m}^{1}(\xi)=\Phi_{k m}^{4}(\xi)=\Phi_{k m}^{c s}(\xi), \quad \Phi_{k m}^{2}(\xi)=\Phi_{k m}^{5}(\xi)=\Phi_{k m}^{s c}(\xi), \quad \Phi_{k m}^{3}(\xi)=\Phi_{k m}^{s s}(\xi), \\
& {\left[\Omega_{k m}^{(U)}(\alpha)\right]=\left[\begin{array}{ccccc}
u_{1 n}(\alpha) & u_{2 n}(\alpha) & u_{3 n}(\alpha) & u_{4 n}(\alpha) & u_{5 n}(\alpha) \\
u_{1 \tau}(\alpha) & u_{2 \tau}(\alpha) & u_{3 \tau}(\alpha) & u_{4 \tau}(\alpha) & u_{5 \tau}(\alpha) \\
w_{1}(\alpha) & w_{2}(\alpha) & w_{3}(\alpha) & w_{4}(\alpha) & w_{5}(\alpha) \\
\gamma_{1 n}(\alpha) & \gamma_{2 n}(\alpha) & \gamma_{3 n}(\alpha) & \gamma_{4 n}(\alpha) & \gamma_{5 n}(\alpha) \\
\gamma_{1 \tau}(\alpha) & \gamma_{2 \tau}(\alpha) & \gamma_{3 \tau}(\alpha) & \gamma_{4 \tau}(\alpha) & \gamma_{5 \tau}(\alpha)
\end{array}\right]} \\
& u_{j n}(\alpha)=n_{1}(\alpha) u_{1 k m}^{j} \Phi_{k m}^{c s}(\alpha)+n_{2}(\alpha) u_{2 k m}^{j} \Phi_{k m}^{s c}(\alpha) \text {, } \\
& u_{j \tau}(\alpha)=\tau_{1}(\alpha) u_{1 k m}^{j} \Phi_{k m}^{c s}(\alpha)+\tau_{2}(\alpha) u_{2 k m}^{j} \Phi_{k m}^{s c}(\alpha), \\
& \gamma_{j n}(\alpha)=n_{1}(\alpha) \gamma_{1 k m}^{j} \Phi_{k m}^{c s}(\alpha)+n_{2}(\alpha) \gamma_{2 k m}^{j} \Phi_{k m}^{s c}(\alpha), \\
& \gamma_{j \tau}(\alpha)=\tau_{1}(\alpha) \gamma_{1 k m}^{j} \Phi_{k m}^{c s}(\alpha)+\tau_{2}(\alpha) \gamma_{2 k m}^{j} \Phi_{k m}^{s c}(\alpha), \\
& w_{j}(\alpha)=w_{k m}^{j} \Phi_{k m}^{s s}(\alpha), \quad j=\overline{1,5}, \\
& {\left[\Omega_{k m}^{(P)}(\alpha)\right]=\left[\begin{array}{lllll}
N_{1 n}(\alpha) & N_{2 n}(\alpha) & N_{3 n}(\alpha) & N_{4 n}(\alpha) & N_{5 n}(\alpha) \\
N_{1 \tau}(\alpha) & N_{2 \tau}(\alpha) & N_{3 \tau}(\alpha) & N_{4 \tau}(\alpha) & N_{5 \tau}(\alpha) \\
Q_{1 n}(\alpha) & Q_{2 n}(\alpha) & Q_{3 n}(\alpha) & Q_{4 n}(\alpha) & Q_{5 n}(\alpha) \\
M_{1 n}(\alpha) & M_{2 n}(\alpha) & M_{3 n}(\alpha) & M_{4 n}(\alpha) & M_{5 n}(\alpha) \\
M_{1 \tau}(\alpha) & M_{2 \tau}(\alpha) & M_{3 \tau}(\alpha) & M_{4 \tau}(\alpha) & M_{5 \tau}(\alpha)
\end{array}\right]} \\
& N_{j n}(\alpha)=B_{1}\left(n_{1}(\alpha)\right)^{2} \Phi_{k m}^{s s}(\alpha)\left[-\lambda_{1 k} u_{1 k m}^{j}-\nu_{12} \lambda_{2 m} u_{2 k m}^{j}+\left(k_{1}+\nu_{12} k_{2}\right) w_{k m}^{j}\right] \\
& +2 B_{12} n_{1}(\alpha) n_{2}(\alpha) \Phi_{k m}^{c c}(\alpha)\left(\lambda_{2 m} u_{1 k m}^{j}+\lambda_{1 k} u_{2 k m}^{j}\right) \\
& +B_{2}\left(n_{2}(\alpha)\right)^{2} \Phi_{k m}^{s s}(\alpha)\left[-\lambda_{2 m} u_{2 k m}^{j}-\nu_{21} \lambda_{1 k} u_{1 k m}^{j}+\left(k_{2}+\nu_{21} k_{1}\right) w_{k m}^{j}\right] \text {, } \\
& N_{j \tau}(\alpha)=B_{1} n_{1}(\alpha) \tau_{1}(\alpha) \Phi_{k m}^{s s}(\alpha)\left[-\lambda_{1 k} u_{1 k m}^{j}-\nu_{12} \lambda_{2 m} u_{2 k m}^{j}+\left(k_{1}+\nu_{12} k_{2}\right) w_{k m}^{j}\right] \\
& +B_{12}\left(n_{1}(\alpha) \tau_{2}(\alpha)+n_{2}(\alpha) \tau_{1}(\alpha)\right) \Phi_{k m}^{c c}(\alpha)\left(\lambda_{2 m} u_{1 k m}^{j}+\lambda_{1 k} u_{2 k m}^{j}\right) \\
& +B_{2} n_{2}(\alpha) \tau_{2}(\alpha) \Phi_{k m}^{s s}(\alpha)\left[-\lambda_{2 m} u_{2 k m}^{j}-\nu_{21} \lambda_{1 k} u_{1 k m}^{j}+\left(k_{2}+\nu_{21} k_{1}\right) w_{k m}^{j}\right] \text {, } \\
& M_{j n}(\alpha)=D_{1}\left(n_{1}(\alpha)\right)^{2} \Phi_{k m}^{s s}(\alpha)\left(-\lambda_{1 k} \gamma_{1 k m}^{j}-\nu_{12} \lambda_{2 m} \gamma_{2 k m}^{j}\right)+2 D_{12} n_{1}(\alpha) n_{2}(\alpha) \Phi_{k m}^{c c}(\alpha) \\
& \times\left(\lambda_{2 m} \gamma_{1 k m}^{j}+\lambda_{1 k} \gamma_{2 k m}^{j}\right)+D_{2}\left(n_{2}(\alpha)\right)^{2} \Phi_{k m}^{s s}(\alpha)\left(-\lambda_{2 m} \gamma_{2 k m}^{j}-\nu_{21} \lambda_{1 k} \gamma_{1 k m}^{j}\right), \\
& M_{j \tau}(\alpha)=D_{1} n_{1}(\alpha) \tau_{1}(\alpha) \Phi_{k m}^{s s}(\alpha)\left(-\lambda_{1 k} \gamma_{1 k m}^{j}-\nu_{12} \lambda_{2 m} \gamma_{2 k m}^{j}\right) \\
& +D_{12}\left(n_{1}(\alpha) \tau_{2}(\alpha)+n_{2}(\alpha) \tau_{1}(\alpha)\right) \Phi_{k m}^{c c}(\alpha)\left(\lambda_{2 m} \gamma_{1 k m}^{j}+\lambda_{1 k} \gamma_{2 k m}^{j}\right) \\
& +D_{2} n_{2}(\alpha) \tau_{2}(\alpha) \Phi_{k m}^{s s}(\alpha)\left(-\lambda_{2 m} \gamma_{2 k m}^{j}-\nu_{21} \lambda_{1 k} \gamma_{1 k m}^{j}\right) \text {, } \\
& Q_{j n}(\alpha)=\Lambda_{1} n_{1}(\alpha) \Phi_{k m}^{c s}(\alpha)\left(\gamma_{1 k m}^{j}+\lambda_{1 k} w_{k m}^{j}-k_{1} u_{1 k m}^{j}\right) \\
& +\Lambda_{2} n_{2}(\alpha) \Phi_{k m}^{s c}(\alpha)\left(\gamma_{2 k m}^{j}+\lambda_{2 m} w_{k m}^{j}-k_{2} u_{2 k m}^{j}\right), \quad j=\overline{1,5} .
\end{aligned}
$$

Mathematical Modeling and Computing, Vol.5, No. 2, pp. 221-234 (2018) 
The solution of system of integral equations can be found using many numerical techniques. For example, the collocation method provides sufficiently good results. The contours of the generalized curve $L$ are approximated by the broken lines $\left(S^{(j)}\right.$ is the the number of linear sections of the $j$-th contour, $\alpha^{(j) r}$ is the middle point of the linear section of the $j$-th contour, $l^{(j) r}$ is the length of the corresponding linear section $\left.L^{(j) r}, r=\overline{1, S^{(j)}}\right)$. The approximation of the unknown density functions on each linear section can be taken as $\left\{T^{(j)}(\xi)\right\}=\left\{T^{(j) r}\right\} \delta\left(\alpha^{(j) r}, \xi\right)$. The residual can be minimized in the number of collocation points $\alpha^{(j) q}$ coinciding with the middle points of the linear sections. Hence, the system of $5 \sum_{j=1}^{N+3} S^{(j)}+N$ linear algebraic equations with the respect to the discrete values of the unknown density functions $\left\{T^{(j) r}\right\}, j=\overline{1, N+3}, r=\overline{1, S^{(j)}}$ and the amplitudes of the displacements of the inclusions $\tilde{w}_{0}^{(j)}, j=\overline{1, N}$ obtains the form

$$
\begin{aligned}
& \left\{u_{n 0}^{(j)}\left(\alpha^{(j) q}\right), u_{\tau 0}^{(j)}\left(\alpha^{(j) q}\right), w_{0}^{(j)}\left(\alpha^{(j) q}\right), \gamma_{n 0}^{(j)}\left(\alpha^{(j) q}\right), \gamma_{\tau 0}^{(j)}\left(\alpha^{(j) q}\right)\right\}^{\mathrm{T}} \\
& =\sum_{f=1}^{N+3} \sum_{r=1}^{S^{(f)}} \sum_{k=0}^{K} \sum_{m=0}^{M} C_{k m}(\varepsilon)\left[\Omega_{k m}^{(U)}\left(\alpha^{(j) q}\right)\right]\left[E_{k m}\left(\alpha^{(f) r}\right)\right]\left\{T^{(f) r}\right\}, \\
& \alpha^{(j) q} \in L^{(j)}, \quad q=\overline{1, S^{(j)}}, \quad j=\overline{N_{1}+1, N_{1}+N_{2}}, \quad j=N+1, \\
& \left\{N_{n 0}^{(j)}\left(\alpha^{(j) q}\right), N_{\tau 0}^{(j)}\left(\alpha^{(j) q}\right), Q_{n 0}^{(j)}\left(\alpha^{(j) q}\right), M_{n 0}^{(j)}\left(\alpha^{(j) q}\right), M_{\tau 0}^{(j)}\left(\alpha^{(j) q}\right)\right\}^{\mathrm{T}} \\
& =\sum_{f=1}^{N+3} \sum_{r=1}^{S^{(f)}} \sum_{k=0}^{K} \sum_{m=0}^{M} C_{k m}(\varepsilon)\left[\Omega_{k m}^{(P)}\left(\alpha^{(j) q}\right)\right]\left[E_{k m}\left(\alpha^{(f) r}\right)\right]\left\{T^{(f) r}\right\}, \\
& \alpha^{(j) q} \in L^{\varepsilon(j)}, \quad q=\overline{1, S^{(j)}}, \quad j=\overline{1, N_{1}}, \quad j=N+2, \\
& w_{0}^{(j)}\left(\alpha^{(j) q}\right)=\sum_{f=1}^{N+3} \sum_{r=1}^{S^{(f)}} \sum_{k=0}^{K} \sum_{m=0}^{M} \sum_{i=1}^{5} C_{k m}(\varepsilon) w_{i}\left(\alpha^{(j) q}\right) \Phi_{k m}^{i}\left(\alpha^{(f) r}\right) T_{i}^{(f) r}, \\
& u_{\tau 0}^{(j)}\left(\alpha^{(j) q}\right)=\sum_{f=1}^{N+3} \sum_{r=1}^{S^{(f)}} \sum_{k=0}^{K} \sum_{m=0}^{M} \sum_{i=1}^{5} C_{k m}(\varepsilon) u_{i \tau}\left(\alpha^{(j) q}\right) \Phi_{k m}^{i}\left(\alpha^{(f) r}\right) T_{i}^{(f) r}, \\
& \gamma_{\tau 0}^{(j)}\left(\alpha^{(j) q}\right)=\sum_{f=1}^{N+3} \sum_{r=1}^{S^{(f)}} \sum_{k=0}^{K} \sum_{m=0}^{M} \sum_{i=1}^{5} C_{k m}(\varepsilon) \gamma_{i \tau}\left(\alpha^{(j) q}\right) \Phi_{k m}^{i}\left(\alpha^{(f) r}\right) T_{i}^{(f) r} \\
& \alpha^{(j) q} \in L^{(j)}, \quad q=\overline{1, S^{(j)}}, \quad j=\overline{N_{1}+N_{2}+1, N}, \quad j=N+3, \\
& M_{n 0}^{(j)}\left(\alpha^{(j) q}\right)=\sum_{f=1}^{N+3} \sum_{r=1}^{S^{(f)}} \sum_{k=0}^{K} \sum_{m=0}^{M} \sum_{i=1}^{5} C_{k m}(\varepsilon) M_{i n}\left(\alpha^{(j) q}\right) \Phi_{k m}^{i}\left(\alpha^{(f) r}\right) T_{i}^{(f) r}, \\
& N_{n 0}^{(j)}\left(\alpha^{(j) q}\right)=\sum_{f=1}^{N+3} \sum_{r=1}^{S^{(f)}} \sum_{k=0}^{K} \sum_{m=0}^{M} \sum_{i=1}^{5} C_{k m}(\varepsilon) N_{i n}\left(\alpha^{(j) q}\right) \Phi_{k m}^{i}\left(\alpha^{(f) r}\right) T_{i}^{(f) r} \\
& \alpha^{(j) q} \in L^{\varepsilon(j)}, \quad q=\overline{1, S^{(j)}}, \quad j=\overline{N_{1}+N_{2}+1, N}, \quad j=N+3,
\end{aligned}
$$




$$
\begin{aligned}
& P_{0}^{(j)}=-\sum_{p=1}^{S^{(j)}} \sum_{f=1}^{N+3} \sum_{r=1}^{S^{(f)}} \sum_{k=0}^{K} \sum_{m=0}^{M} \sum_{i=1}^{5} C_{k m}(\varepsilon) \Psi_{k m}^{s s}\left(\alpha^{(j) p}\right) w_{k m}^{i} \Phi_{k m}^{i}\left(\alpha^{(f) r}\right) T_{i}^{(f) r}++\tilde{w}_{0}^{(j)}\left(\sum_{p=1}^{S^{(j)}} \Theta\left(\alpha^{(j) p}\right)-\omega^{2} \tilde{m}^{(j)}\right), \quad j=\overline{1, N_{1}}, \\
& P_{0}^{(j)}=\sum_{p=1}^{S^{(j)}} \sum_{f=1}^{N+3} \sum_{r=1}^{S^{(f)}} \sum_{k=0}^{K} \sum_{m=0}^{M} \sum_{i=1}^{5} C_{k m}(\varepsilon) \Psi_{i n}\left(\alpha^{(j) p}\right) \Phi_{k m}^{i}\left(\alpha^{(f) r}\right) T_{i}^{(f) r}-\omega^{2} \tilde{m}^{(j)} \tilde{w}_{0}^{(j)}, \\
& j=\overline{N_{1}+1, N_{1}+N_{2}+N_{3}}
\end{aligned}
$$

where

$$
\begin{gathered}
u_{n 0}^{(j)}\left(\alpha^{(j) q}\right)=u_{\tau 0}^{(j)}\left(\alpha^{(j) q}\right)=\gamma_{n 0}^{(j)}\left(\alpha^{(j) q}\right)=\gamma_{\tau 0}^{(j)}\left(\alpha^{(j) q}\right)=0, \quad w_{0}^{(j)}\left(\alpha^{(j) q}\right)=\tilde{w}_{0}^{(j)}, \quad j=\overline{N_{1}+1, N_{1}+N_{2}}, \\
N_{n 0}^{(j)}\left(\alpha^{(j) q}\right)=N_{\tau 0}^{(j)}\left(\alpha^{(j) q}\right)=M_{n 0}^{(j)}\left(\alpha^{(j) q}\right)=M_{\tau 0}^{(j)}\left(\alpha^{(j) q}\right)=0, \\
Q_{n 0}^{(j)}\left(\alpha^{(j) q}\right)=k^{(j)}\left(\alpha^{(j) q}\right)\left(\tilde{w}_{0}^{(j)}-\sum_{f=1}^{N+3} \sum_{r=1}^{S^{(f)}} \sum_{k=0}^{K} \sum_{m=0}^{M} \sum_{i=1}^{5} C_{k m}(\varepsilon) w_{i}\left(\alpha^{(j) q}\right) \Phi_{k m}^{i}\left(\alpha^{(f) r}\right) T_{i}^{(f) r}\right), \quad j=\overline{1, N_{1}}, \\
N_{n 0}^{(j)}\left(\alpha^{(j) q}\right)=u_{\tau 0}^{(j)}\left(\alpha^{(j) q}\right)=M_{n 0}^{(j)}\left(\alpha^{(j) q}\right)=\gamma_{\tau 0}^{(j)}\left(\alpha^{(j) q}\right)=0, \quad w_{0}^{(j)}\left(\alpha^{(j) q}\right)=\tilde{w}_{0}^{(j)}, \\
\Psi_{i n}\left(\alpha^{(j) p}\right)=\Lambda_{1} \Psi_{k m}^{c s}\left(\alpha^{(j) p}\right)\left(\gamma_{1 k m}^{i}+\lambda_{1 k} w_{k m}^{i}-k_{1} u_{1 k m}^{i}\right)+\Lambda_{2} \Psi_{k m}^{s c}\left(\alpha^{(j) p}\right)\left(\gamma_{2 k m}^{i}+\lambda_{2 m} w_{k m}^{i}-k_{2} u_{2 k m}^{i}\right), \\
\Psi_{k m}^{c s}\left(\alpha^{(j) p}\right)=n_{1}\left(\alpha^{(j) p}\right) \int_{L^{\varepsilon(j) p}} \Phi_{k m}^{c s}(\zeta) d l(\zeta), \\
\Psi_{k m}^{s c}\left(\alpha^{(j) p}\right)=n_{2}\left(\alpha^{(j) p}\right) \int_{L^{\varepsilon(j) p}} \Phi_{k m}^{s c}(\zeta) d l(\zeta), \quad \alpha^{(j) p} \in L^{\varepsilon(j) p}, \\
\Psi_{k m}^{s s}\left(\alpha^{(j) p}\right)=\int_{L^{(j) p}} k^{(j)}(\zeta) \Phi_{k m}^{s s}(\zeta) d l(\zeta), \quad \Theta\left(\alpha^{(j) p}\right)=\int_{L^{(j) p}} k^{(j)}(\zeta) d l(\zeta), \quad \alpha^{(j) p} \in L^{(j) p} .
\end{gathered}
$$

The natural frequencies can be found by equating the determinant of the system of linear algebraic equations with zero. On the basis of the discrete values of the density functions of the simple layer potential the characteristics of the stress-strain state along arbitrary direction with the normal vector $\boldsymbol{n}(\alpha)$ and tangential vector $\boldsymbol{\tau}(\alpha)$ can be calculated using the following formulas:

$$
\begin{aligned}
& \left\{\begin{array}{c}
u_{n}(\alpha, t) \\
u_{\tau}(\alpha, t) \\
w(\alpha, t) \\
\gamma_{n}(\alpha, t) \\
\gamma_{\tau}(\alpha, t)
\end{array}\right\}=\sum_{j=1}^{N+3} \sum_{r=1}^{S^{(j)}} \sum_{k=0}^{K} \sum_{m=0}^{M} C_{k m}(\varepsilon)\left[\Omega_{k m}^{(U)}(\alpha)\right]\left[E_{k m}\left(\alpha^{(j) r}\right)\right]\left\{T^{(j) r}\right\} \sin (\omega t), \\
& \left\{\begin{array}{c}
N_{n}(\alpha, t) \\
N_{\tau}(\alpha, t) \\
Q_{n}(\alpha, t) \\
M_{n}(\alpha, t) \\
M_{\tau}(\alpha, t)
\end{array}\right\}=\sum_{j=1}^{N+3} \sum_{r=1}^{S^{(j)}} \sum_{k=0}^{K} \sum_{m=0}^{M} C_{k m}(\varepsilon)\left[\Omega_{k m}^{(P)}(\alpha)\right]\left[E_{k m}\left(\alpha^{(j) r}\right)\right]\left\{T^{(j) r}\right\} \sin (\omega t) .
\end{aligned}
$$

Mathematical Modeling and Computing, Vol.5, No. 2, pp. 221-234 (2018) 


\section{Numerical results}

Using the algebraic equations constructed in the paper one can consider different partial cases of the problem. For the verification of the numerical scheme, the partial case of simply supported rectangular in plane doubly curved panel with two circular rigidly connected to the panel inclusions is considered. In this case it is reasonable to choose the domain $\Pi$ in the way that its boundary coincides with the external boundary of the panel. The geometrical and physical parameters of the panel are: $l_{1}=2 \mathrm{~m}$, $l_{2}=1 \mathrm{~m}, 2 h=0.02 \mathrm{~m}, k_{1}=0.1 \mathrm{~m}^{-1}, k_{2}=0.2 \mathrm{~m}^{-1}, G_{12}=G_{13}=4.48 \mathrm{GPa}, G_{23}=1.53 \mathrm{GPa}$, $E_{1}=128 \mathrm{GPa}, E_{2}=11 \mathrm{GPa}, \nu_{12}=0.25, \rho=1500 \frac{\mathrm{kg}}{\mathrm{m}^{3}}$. The masses of the inclusions are taken as: $\tilde{m}^{(1)}=\tilde{m}^{(2)}=100 \mathrm{~kg}$. External forces acting on the inclusions have the values $P_{0}^{(1)}=-P_{0}^{(2)}=50000 \mathrm{~N}$. The radii and locations of the centers of the inclusions are: $b_{1}=0.2 \mathrm{~m}, \alpha_{1}^{*(1)}=0.5 \mathrm{~m}, \alpha_{2}^{*(1)}=0.5 \mathrm{~m}$; $b_{2}=0.2 \mathrm{~m}, \alpha_{1}^{*(2)}=1.5 \mathrm{~m}, \alpha_{2}^{*(2)}=0.5 \mathrm{~m}$.

The boundary conditions on the simply supported external boundary of the panel have the form:

$$
w(\alpha, t)=0, \quad u_{\tau}(\alpha, t)=0, \quad \gamma_{\tau}(\alpha, t)=0, \quad M_{n}(\alpha, t)=0, \quad N_{n}(\alpha, t)=0, \quad \alpha \in L^{(3)} .
$$

The parameters of approximation are as follows: $K=50, S^{(1)}=S^{(2)}=20, \varepsilon_{1}=\varepsilon_{2}=\varepsilon=\frac{b_{2} \pi}{S^{(2)}} \mathrm{m}$. Such method of generalized summation is used:

$$
C_{k m}(\varepsilon)=\mu_{k m} \frac{4}{l_{1} l_{2}} \frac{\sin ^{2} \frac{\lambda_{1 k} \varepsilon_{1}}{2} \sin ^{2} \frac{\lambda_{2 m} \varepsilon_{2}}{2}}{\left(\frac{\lambda_{1 k} \varepsilon_{1}}{2}\right)^{2}\left(\frac{\lambda_{2 m} \varepsilon_{2}}{2}\right)^{2}} .
$$

In the cases, when $k=0$ or $m=0$, the well known limit $\lim _{x \rightarrow 0} \frac{\sin x}{x}=1$ is used for calculating the value of the expression $C_{k m}(\varepsilon)$.

Parametric representation of the contours of the inclusions with the proper choice of the orientation of the normal and tangential vectors to the contours of the inclusions is used. Hence, we obtain

$$
\begin{gathered}
\alpha_{1}^{(j) q}=\alpha_{1}^{*(j)}+b_{j} \cos \frac{2 \pi q}{S^{(j)}}, \quad \alpha_{2}^{(j) q}=\alpha_{2}^{*(j)}-b_{j} \sin \frac{2 \pi q}{S^{(j)}}, \\
n_{1}\left(\alpha^{(j) q}\right)=-\cos \frac{2 \pi q}{S^{(j)}}, \quad n_{2}\left(\alpha^{(j) q}\right)=\sin \frac{2 \pi q}{S^{(j)}}, \quad \tau_{1}\left(\alpha^{(j) q}\right)=-n_{2}\left(\alpha^{(j) q}\right), \quad \tau_{2}\left(\alpha^{(j) q}\right)=n_{1}\left(\alpha^{(j) q}\right), \\
\alpha_{1}^{(j) p}=\alpha_{1}^{*(j)}+\left(b_{j}+\varepsilon\right) \cos \frac{2 \pi p}{S^{(j)}}, \quad \alpha_{2}^{(j) p}=\alpha_{2}^{*(j)}-\left(b_{j}+\varepsilon\right) \sin \frac{2 \pi p}{S^{(j)}}, \\
\alpha_{1}^{(j) r}=\alpha_{1}^{*(j)}+b_{j} \cos \frac{2 \pi r}{S^{(j)}}, \quad \alpha_{2}^{(j) r}=\alpha_{2}^{*(j)}-b_{j} \sin \frac{2 \pi r}{S^{(j)}}, \quad j=1,2 .
\end{gathered}
$$

In Fig. 1, the deflection amplitude of the panel for different values of the inclusions' radii under the vibration frequency $\varpi=\frac{\omega}{2 \pi}=35 \mathrm{~Hz}$ is shown. It can be seen that, as the inclusions' radii increase, the deflection amplitude of the panel and the amplitude of the displacements of the inclusions decrease.

In Fig. 2 and Fig. 3, the amplitude of deflection of the panel for different values of the masses of the inclusions and for different values of the vibration frequency is presented. The results show that in these cases the simplest form of the vibration takes place. Under the relatively low frequency of vibration moderate increase of the masses of the inclusions leads to the increase of the amplitude of the deflection of the panel and the displacements of the inclusions (Fig. 2). However, for the cases of higher frequencies the opposite effect can be observed (Fig. 3). So on the whole, one can conclude that there is generally non monotonic relationship between the amplitude of deflection of the panel or the displacements of the inclusions and masses of the inclusions.

In Fig. 4 and Fig. 5, the amplitude of the deflection of the panel for different values of the vibration frequency is shown. In both cases the simplest form of vibration takes place and the vibration frequency substantially influences the amplitude of the deflection of the panel and the displacements of the inclusions. On the lower frequency range the sharp increase of the amplitude of the deflection of the 


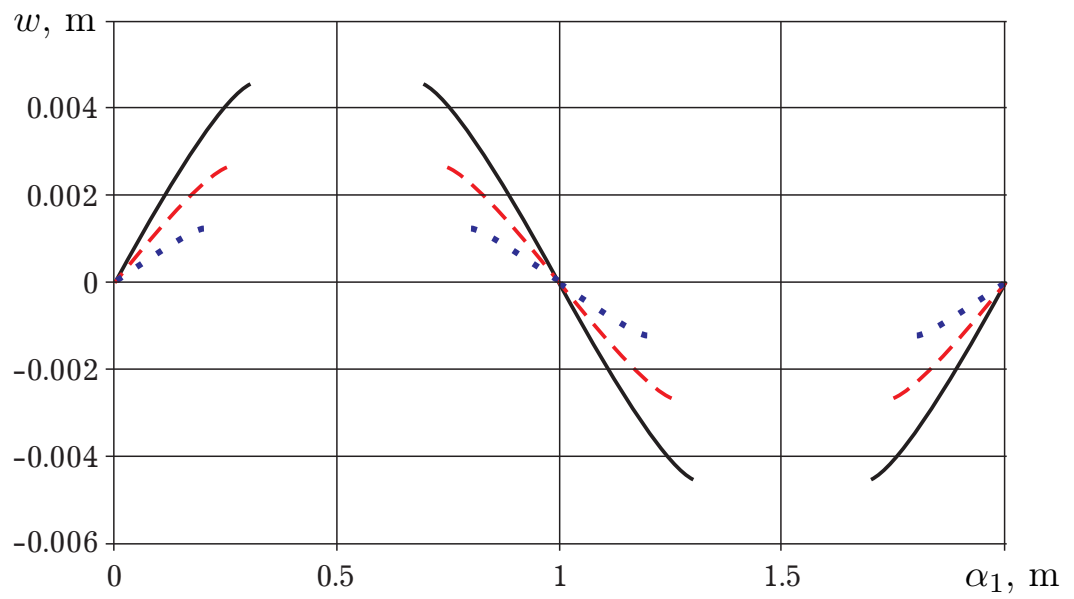

Fig. 1. The amplitude of deflection of the panel on the line $\alpha_{2}=0.5 \mathrm{~m}$ for different values of the inclusions' radii: solid curve for $b_{1}=0.2 \mathrm{~m}, b_{2}=0.2 \mathrm{~m}$, dashed curve for $b_{1}=0.25 \mathrm{~m}$, $b_{2}=0.25 \mathrm{~m}$, dotted curve for $b_{1}=0.3 \mathrm{~m}, b_{2}=0.3 \mathrm{~m}$.

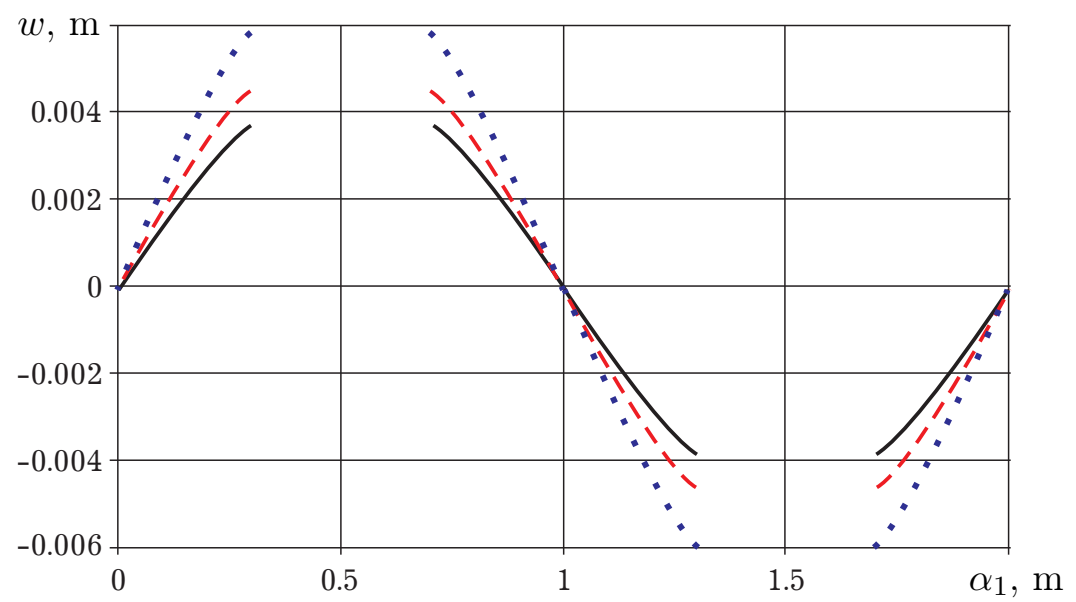

Fig. 2. The amplitude of deflection of the panel on the line $\alpha_{2}=0.5 \mathrm{~m}$ for different values of the inclusions' masses under the frequency $\varpi=\frac{\omega}{2 \pi}=35 \mathrm{~Hz}$ : solid curve for $\tilde{m}^{(1)}=\tilde{m}^{(2)}=50 \mathrm{~kg}$, dashed curve for $\tilde{m}^{(1)}=\tilde{m}^{(2)}=100 \mathrm{~kg}$, dotted curve for $\tilde{m}^{(1)}=\tilde{m}^{(2)}=150 \mathrm{~kg}$.

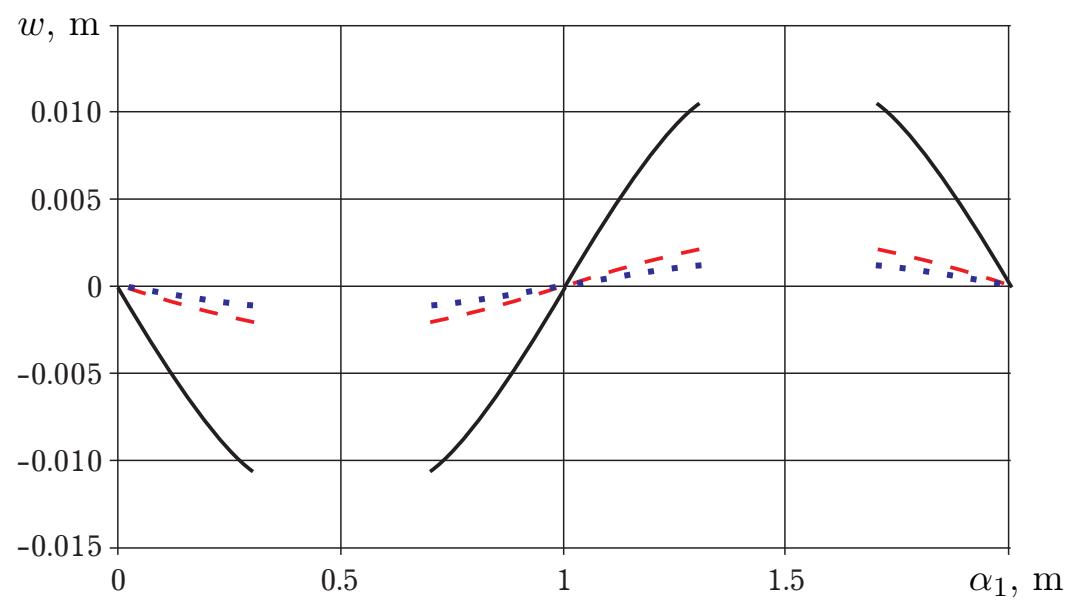

Fig. 3. Deflection amplitude of the panel on the line $\alpha_{2}=0.5 \mathrm{~m}$ for different values of inclusions' masses under the vibration frequency $\varpi=\frac{\omega}{2 \pi}=100 \mathrm{~Hz}$ : solid curve for $\tilde{m}^{(1)}=\tilde{m}^{(2)}=50 \mathrm{~kg}$, dashed curve for $\tilde{m}^{(1)}=\tilde{m}^{(2)}=100 \mathrm{~kg}$, dotted curve for $\tilde{m}^{(1)}=\tilde{m}^{(2)}=150 \mathrm{~kg}$. 
panel and the displacements of the inclusions under the increasing vibration frequency can be observed. On the contrast, on the higher frequency range the amplitude of the panel and displacements of the inclusions tend to decrease. Consequently, there is non monotonic relationship between the amplitude and vibration frequency. Thus, not only the natural frequencies are dangerous for the construction, the low frequencies before the first resonance occurs can be also dangerous.

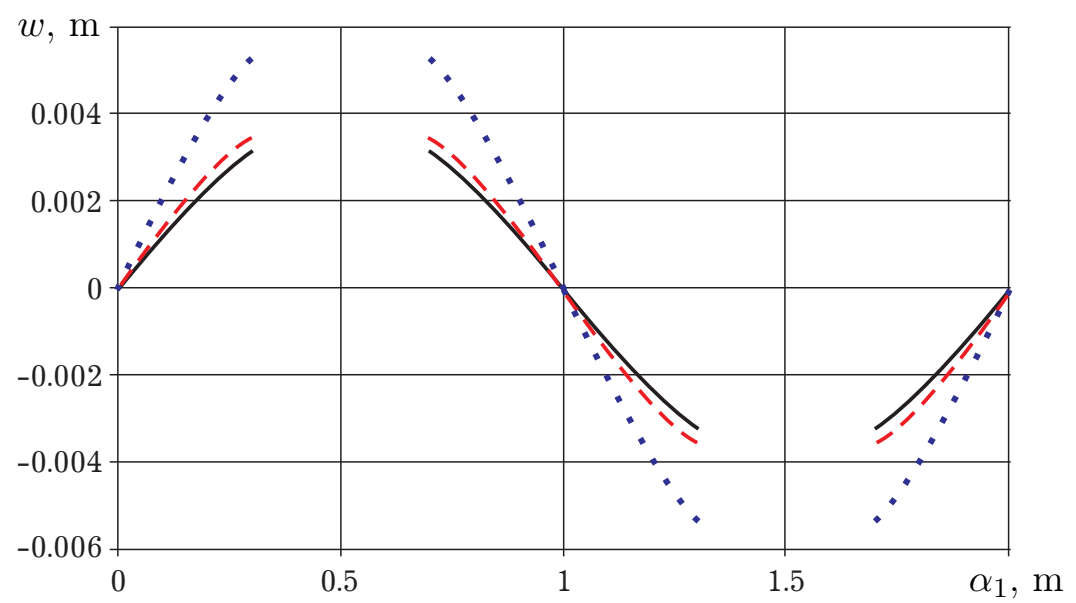

Fig. 4. The amplitude of deflection of the panel on the line $\alpha_{2}=0.5 \mathrm{~m}$ for different values of the vibration frequency $\varpi=\frac{\omega}{2 \pi}$ : solid curve for $\varpi=5 \mathrm{~Hz}$, dashed curve for $\varpi=20 \mathrm{~Hz}$, dotted curve for $\varpi=40 \mathrm{~Hz}$.

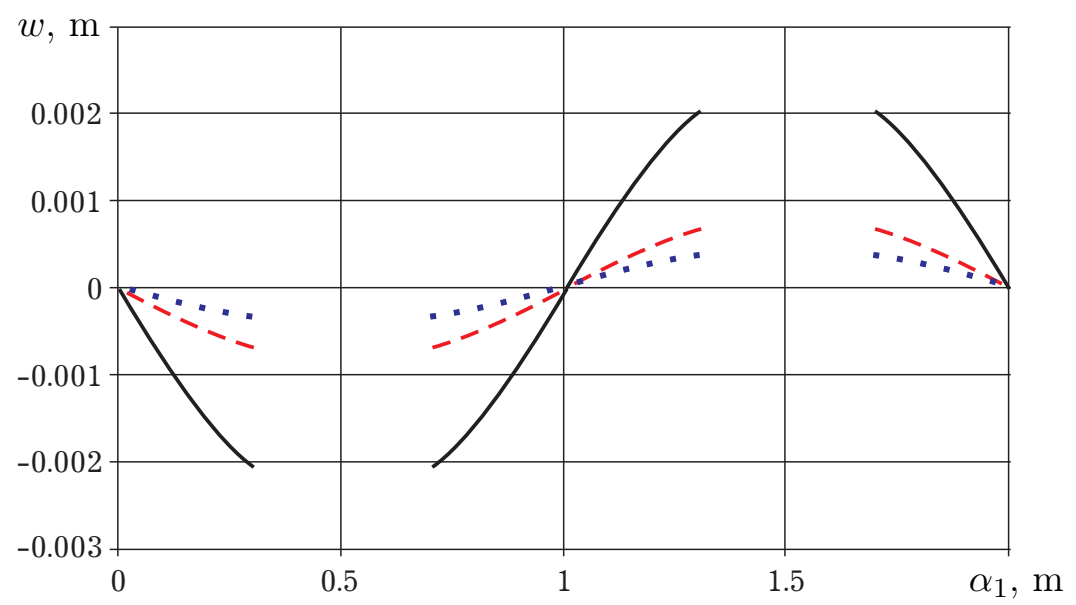

Fig. 5. The amplitude of deflection of the panel on the line $\alpha_{2}=0.5 \mathrm{~m}$ for different values of the vibration frequency $\varpi=\frac{\omega}{2 \pi}$ : solid curve for $\varpi=100 \mathrm{~Hz}$, dashed curve for $\varpi=150 \mathrm{~Hz}$, dotted curve for $\varpi=200 \mathrm{~Hz}$.

In Fig. 6, the non-symmetrical case is considered, when inclusions have different masses $\tilde{m}^{(1)}=50 \mathrm{~kg}, \tilde{m}^{(2)}=100 \mathrm{~kg}$, are subjected to different external forces $P_{0}^{(1)}=35000, P_{0}^{(2)}=-65000$, have different radii $b_{1}=0.1 \mathrm{~m}, b_{2}=0.2 \mathrm{~m}$ and are located non-symmetrically. The influence of location of the second inclusion on the deflection amplitude of the panel under the vibration frequency $\varpi=\frac{\omega}{2 \pi}=35 \mathrm{~Hz}$ is shown. The coordinates of the first inclusion are taken as $\alpha_{1}^{*(1)}=0.4 \mathrm{~m}$, $\alpha_{2}^{*(1)}=0.5 \mathrm{~m}$.

Fig. 6 demonstrates that the change of the relative location of the inclusions influences the deflection amplitude of the panel on all sections and influences the displacement amplitudes of both inclusions. 


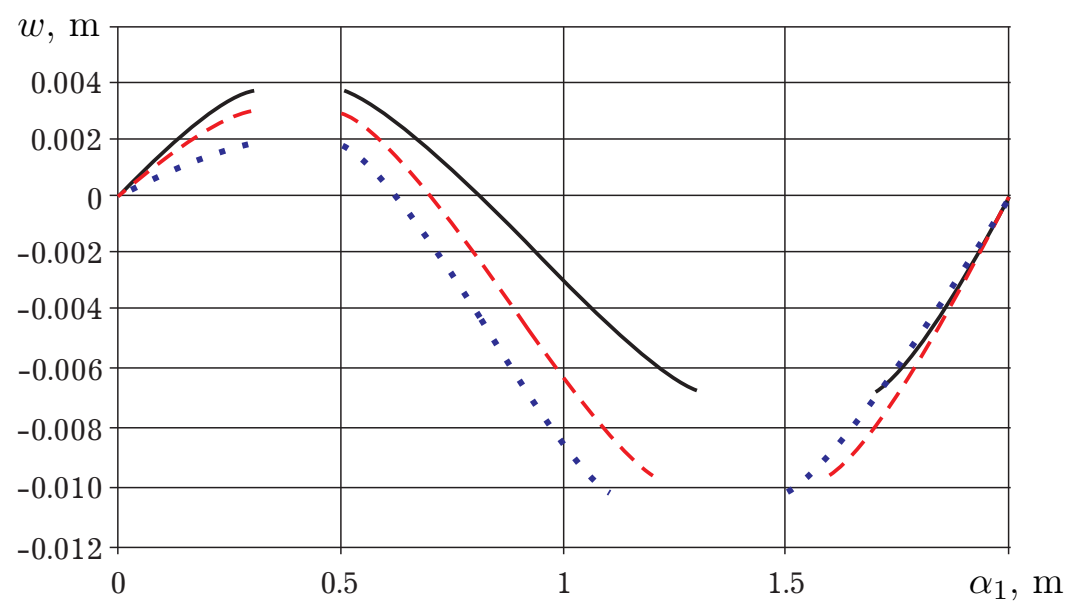

Fig. 6. Deflection amplitude of the panel on the line $\alpha_{2}=0.5 \mathrm{~m}$ in the case of different location of the second inclusion: solid curve for $\alpha_{1}^{*(2)}=1.5 \mathrm{~m}, \alpha_{2}^{*(2)}=0.5 \mathrm{~m}$, dashed curve for $\alpha_{1}^{*(2)}=1.4 \mathrm{~m}, \alpha_{2}^{*(2)}=0.5 \mathrm{~m}$, dotted curve for $\alpha_{1}^{*(2)}=1.3 \mathrm{~m}, \alpha_{2}^{*(2)}=0.5 \mathrm{~m}$.

\section{Conclusions}

The obtained numerical results show that the dynamic deflection of the panel depends on the frequency of the vibration, mass, size, and the location of the inclusions substantially. Thus, not only the natural frequencies are dangerous for the construction, the low frequencies before the first resonance occurs can be also dangerous. The constructed solutions allow us to make the efficient choice of the shape, location, mass of the inclusions, different physical and geometrical parameters of the panel for providing the reliability of the construction under the dynamic loads. The generalized numerical scheme presented in the paper can be used for the investigation of the solutions for different partial cases of the problem.

[1] Mykhas'kiv V., Kunets Ya., Matus V., Khay O. Elastic wave dispersion and attenuation caused by multiple types of disc-shaped inclusions. International Journal of Structural Integrity. 9 (2), 219-232 (2018).

[2] Kit H. S., Mykhas'skiv V. V., Khaj O. M. Analysis of the steady oscillations of a plane absolutely rigid inclusion in a three-dimensional elastic body by the boundary element method. Journal of applied mathematics and mechanics. 66 (5), 817-824 (2002).

[3] Mykhas'kiv V. V., Khay O. M., Zhang C., Boström A. Effective dynamic properties of 3D composite materials containing rigid penny-shaped inclusions. Waves in Random and Complex Media. 20 (3), 491-510 (2010).

[4] Mykhas'kiv V. Transient response of a plane rigid inclusion to an incident wave in an elastic solid. Wave motion. 41 (2), 133-144 (2005).

[5] Kit G. S., Kunets Ya. I., Mykhas'kiv V. V. Interaction of a stationary wave with a thin low stiffness pennyshaped inclusion in an elastic body. Mechanics of solids. 39 (5), 64-70 (2004).

[6] Burak Ja. J., Rudavsky Ju. K., Sukhorolsky M. A. Analitychna mechanika lokalno navantazhenyh obolonok. Lviv, Intelekt-Zakhid (2007), (in Ukrainian).

[7] Shopa T. Do pobudovy rozvazku zadachi pro kolyvanna ortotropnoi nepolohoji zylindrychnoi paneli z vkluchennam dovilnoi konfigurazii. Mashynoznavstvo. 7, 38-42 (2010), (in Ukrainian).

[8] Shopa T. Kolyvanna ortotropnoi paneli podvijnoi kryvyny z mnozhynoju vkluchen dovilnoi konfihurazii. Suchasni problemy mechaniky ta matematyky. 2, 187-188 (2013), (in Ukrainian).

[9] Shopa T. Kolyvanna ortotropnoi paneli podvijnoi kryvyny z mnozhynoju vkluchen dovilnoi konfihurazii z pruzhnymy prosharkamy. Visnyk Ternopilskoho nazionalnonho tekhnichnoho universytety. 1, 71-84 (2013), (in Ukrainian). 
[10] Shopa T. Kolyvanna ortotropnoi paneli podvijnoi kryvyny z mnozhynoju sharnirno opertyh vkluchen dovilnoi konfihurazii. Prykarpatskij visnyk naukovoho tovarystva Shevchenka. 2, 114-121 (2017), (in Ukrainian).

[11] Shopa T. Kolyvanna ortotropnoi paneli podvijnoi kryvyny z mnozhynoju otvoriv dovilnoji konfihurazii. Visnyk Ternopilskoho nazionalnonho tekhnichnoho universytety. 3, 63-74 (2012), (in Ukrainian).

[12] LighthillJ. Introduction to Fourier Analysis and Generalised Functions. Cambridge University Press (1958).

[13] Sukhorolsky M. A. Funkzionalni poslidovnosti i rady. Lviv, Rastr-7 (2010), (in Ukrainian).

\title{
Коливання ортотропної панелі подвійної кривини з множиною включень довільної конфігурації та різними типами з'єднань з панеллю
}

\author{
Шопа T. \\ Інститут прикладних проблем механіки $і$ математики \\ ім. Я. С. Підстригача НАН України, \\ вул. Наукова, 3-б, Лъвів, 79060, Україна
}

\begin{abstract}
У межах уточненої теорії оболонок, яка враховує поперечні зсуви та всі інерційні компоненти, побудовано розв'язок задачі про усталені коливання ортотропної панелі подвійної кривини з довільною кількістю абсолютно жорстких включень довільної форми та розташування. Включення мають різні типи з'єднань з панеллю і здійснюють поступальний рух вздовж нормального напрямку до серединної поверхні панелі. Зовнішня границя панелі довільної геометричної конфігурації. Розглянуто довільні мішані гармонічні в часі граничні умови на зовнішній границі панелі. Розв'язок побудовано на основі непрямого методу граничних елементів. Використано послідовнісний підхід до подання функцій Гріна. Інтегральні рівняння розв'язано методом колокацій.
\end{abstract}

Ключові слова: ортотропна панель подвійної кривини, включення, коливання, непрямий метод граничних елементів.

2000 MSC: $74 \mathrm{H} 45$

УдК: 539.3 